EPJ Web of Conferences 52, 01004 (2013)

DOI: $10.1051 /$ epjconf/20135201004

(C) Owned by the authors, published by EDP Sciences, 2013

\title{
Results from ALICE
}

Jan Fiete Grosse-Oetringhaus, for the ALICE collaboration ${ }^{1, a}$

${ }^{1}$ CERN

\begin{abstract}
ALICE, the dedicated heavy-ion experiment at the LHC, has also a rich pp program benefiting from its low material budget, low magnetic field, and its extensive particle identification capabilities. The talk presents recent measurements of relevance for cosmic-ray physics. In particular, ALICE measured the inelastic, single- and doublediffractive cross-sections in pp collisions at $\sqrt{s}=0.9,2.76$, and $7 \mathrm{TeV}$ [1]. Further, the charged-particle pseudorapidity distribution $\left(d N_{\mathrm{ch}} / d \eta\right)$ in $\mathrm{Pb}-\mathrm{Pb}$ collisions at $\sqrt{s_{\mathrm{NN}}}=$ $2.76 \mathrm{TeV}$ was measured over a large pseudorapidity range $(-5.0<\eta<5.5)$ using collisions from displaced vertices [2]. Potential direct contributions of ALICE to cosmic-ray physics are also reviewed [3].
\end{abstract}

\section{References}

[1] ALICE Collaboration, "Measurement of inelastic, single- and double-diffraction cross sections in proton-proton collisions at the LHC with ALICE", arXiv:1208.4968

[2] ALICE Collaboration, "Centrality dependence of the pseudorapidity density distribution for charged particles in $\mathrm{Pb}-\mathrm{Pb}$ collisions at $\sqrt{s_{\mathrm{NN}}}=2.76 \mathrm{TeV}$ ”, arXiv:1304.0347

[3] CERN Courier, ALICE looks to the skies, 18.07.12, http://cerncourier.com/cws/article/cern/50219

Slides

The slides of the talk can be found on the website of the symposium ISVHECRI 2012:

https://indico.desy.de/conferenceOtherViews.py?view=standard\&confId=4594

\footnotetext{
ajgrosseo@mail.cern.ch
} 